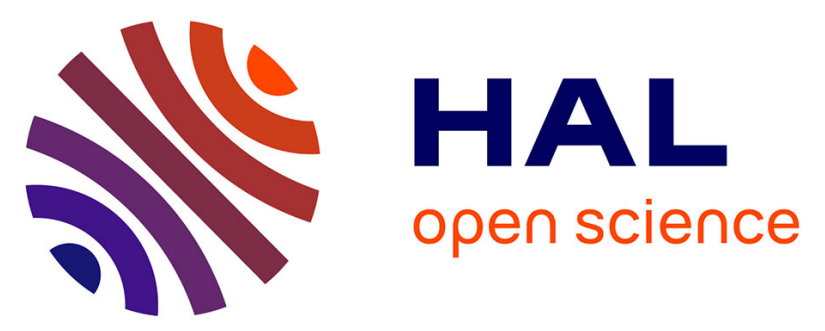

\title{
Etat structural, enracinement et alimentation hydrique du maïs. I. - Modélisation d'états structuraux types de la couche labourée
}

\author{
François Tardieu, Hubert Manichon
}

\section{- To cite this version:}

François Tardieu, Hubert Manichon. Etat structural, enracinement et alimentation hydrique du maïs. I. - Modélisation d'états structuraux types de la couche labourée. Agronomie, 1987, 7 (2), pp.123-131. hal-00884976

\section{HAL Id: hal-00884976 https://hal.science/hal-00884976}

Submitted on 1 Jan 1987

HAL is a multi-disciplinary open access archive for the deposit and dissemination of scientific research documents, whether they are published or not. The documents may come from teaching and research institutions in France or abroad, or from public or private research centers.
L'archive ouverte pluridisciplinaire HAL, est destinée au dépôt et à la diffusion de documents scientifiques de niveau recherche, publiés ou non, émanant des établissements d'enseignement et de recherche français ou étrangers, des laboratoires publics ou privés. 


\title{
Etat structural, enracinement et alimentation hydrique du maïs. I. - Modélisation d'états structuraux types de la couche labourée
}

\author{
François TARDIEU \& Hubert MANICHON $\left({ }^{*}\right)$ \\ I.N.R.A., Laboratoire d'Agronomie INA-PG, Centre de Recherches de Paris-Grignon, F 78850 Thiverval- \\ Grignon \\ (*) INA-PG, Chaire d'Agronomie, F 78850 Thiverval-Grignon
}

RÉSUMÉ

\begin{abstract}
Trois états structuraux types de la couche labourée, qui simulent des situations fréquemment observées en parcelles cultivées, ont été reproduits à 4 reprises sur des dispositifs expérimentaux en sols limoneux à l'aide de techniques appropriées. Ils ont été caractérisés chaque année par des cartes structurales répétées et par des mesures de masse volumique et d'humidité du sol. Le premier, " $O$ », est formé de terre fine et de mottes centimétriques ; il représente des parcelles peu dégradées dans leur histoire récente. Le deuxième, « B », est une juxtaposition de blocs tassés et de cavités structurales, et représente les situations où de forts tassements ont eu lieu avant le labour. Le troisième, « $\mathrm{C}$ », est continu et compact sur $30 \mathrm{~cm}$ au semis, mais non tassé dans les couches non travaillées ; il est le seul où les fentes de retrait soient apparues en cours de saison. Il représente des parcelles où des tassements ont eu lieu après le labour.

Ces essais montrent que : (1) ces états ont pu être obtenus au semis sur chacun des champs expérimentaux ; (2) ils sont reproductibles entre années et entre sites ; (3) la variabilité spatiale de l'état structural à l'intérieur d'une parcelle expérimentale est, à l'échelle métrique, très inférieure à la variabilité inter-parcelles expérimentales. Il est donc possible de considérer les états $\mathrm{O}$, B et $\mathrm{C}$ comme des traitements dans une étude multilocale des conséquences du travail du sol sur les peuplements végétaux.
\end{abstract}

Mots clés additionnels : Variabilité spatiale, tassement du sol, labour, cartes de l'état structural.

\begin{abstract}
Soil structure, root system and water uptake of maize. I. - Modelling of typical ploughed layer structures.

Three types of ploughed layer structure, modelling situations often observed in cultivated fields, were reproduced 4 times in experimental loamy fields, using appropriate techniques. These were characterized each year by structural mapping and measurement of dry bulk density and soil water content. The first, " $\mathrm{O}$ ", is fragmentary, composed of fine earth and $\mathrm{cm}$-sized crumbs; it represents in fields which were not compacted recently. The second, «B $»$, is a juxtaposition of compacted dm-sized blocks and holes; it represents fields where compaction occurred before ploughing. The third, «C », is uniformly compacted to $30 \mathrm{~cm}$ at sowing, but the non-tilled layers are not modified; it is the only one where appreciable cracks appear during the season and relates to fields compacted after ploughing. Results showed that : (1) these structures could be obtained at sowing in each of the experimental fields ; (2) they were reproducible over several fields and years ; (3) the spatial variability of the ploughed layer structure within experimental plots was, at the metre scale, much lower than inter-plot variability. It was thus possible to consider O, B and C typical structures as experimental treatments in
\end{abstract} a multilocational study of the consequences of soil tillage on crops.

Additional key words : Spatial variability, soil compaction, ploughing, soil structure mapping.

\section{INTRODUCTION}

Les expérimentations sur le travail du sol consistent généralement à comparer entre elles des parcelles cultivées avec des outils différents, affectant à celles qui le sont avec les techniques communément utilisées par les agriculteurs la fonction de "témoin ». Pour la culture du maïs, le labour tient le plus souvent ce rôle (BARBER, 1971 ; BODET et al., 1976 ; ElLIS et al., 1978). C'est ainsi que BoIFFIN et al. (1976) ont pu constater, en moyenne, la supériorité vis-à-vis du rendement des techniques comportant un labour, par rapport au semis direct. Les différences de rendement sont cependant très variables suivant les années. Outre les effets clima- 
tiques annuels, on peut voir ici l'influence de la variabilité des états créés dans les parcelles expérimentales labourées (MANICHON \& BODET, 1976). Suivant les années, plusieurs types d'états structuraux de la couche labourée sont créés, mais leurs effets sur les peuplements végétaux ne peuvent être comparés directement entre eux, puisqu'ils sont apparus lors d'années différentes. C'est cette comparaison que nous avons entreprise ici.

Afin d'analyser les relations particulièrement complexes entre le travail du sol et le fonctionnement des peuplements végétaux, il est nécessaire (SEBILlotTe, 1978) de distinguer 2 étapes : (i) l'étude des modifications de l'état du profil cultural (HENIN et al., 1969) sous l'action des outils, et (ii) l'étude de l'effet de cet état sur le peuplement végétal, en interaction avec les conditions climatiques et le comportement du sol considéré. Le travail qui fait l'objet de cette série d'articles a pour but de contribuer à la seconde étude.

Une condition nécessaire pour que celle-ci soit réalisable est qu'on puisse mettre en comparaison dans un même dispositif expérimental plusieurs types d'états structuraux du sol, qui sont en général observés dans des parcelles cultivées distinctes ou lors d'années différentes. Chacun de ces états structuraux modélise alors une situation culturale. Des travaux antérieurs (MANICHON \& SEBILlOTTE, 1975 ; MANICHON 1982a) ont permis d'en définir 3 types, qui recouvrent une gamme assez large des états rencontrés au champ dans la couche labourée : le premier noté «O $O$ », est fragmenté et ne comporte ni obstacles de grande dimension, ni cavités ; le deuxième, noté " $\mathrm{B}$ », comporte des blocs de plusieurs $\mathrm{dm}^{3}$, tassés et séparés par des cavités ; le troisième, noté " $\mathrm{C} »$, est continu et compact. Pour restreindre le facteur de variation du dispositif expérimental à l'état de la seule couche labourée, les profils culturaux que nous avons cherché à modéliser ne sont différents ni dans les couches non travaillées, ni dans le lit de semence (horizon appelé $\mathrm{H} 1$ dans la suite du texte). Ils sont donc définis par l'état structural de la partie de la couche labourée qui n'a pas été affinée lors du travail superficiel postérieur au labour (horizon appelé H5 dans la suite du texte).
Le $1^{\text {er }}$ article de cette série a pour objectif de montrer que la modélisation en champ expérimental de ces 3 états est possible. L'effet de ceux-ci sur la croissance et la disposition spatiale des racines ainsi que les conséquences sur l'alimentation hydrique et la croissance du maïs feront l'objet des articles suivants.

\section{MATÉRIEL ET MÉTHODES}

\section{A. Conditions expérimentales}

Les essais ont été conduits dans la région parisienne, à Grignon (Yvelines) dans les parcelles 1 en 1982, 2 en 1983 et 1985, et à Montmirail (Marne) en 1982. Les 2 parcelles de Grignon sont pédologiquement voisines, celle de Montmirail se distingue (tabl. 1) par un drainage interne déficient, dû à la nature du sous-sol. D'après les relations établies par MONNIER \& STENGEL (1982), la stabilité structurale, liée au taux d'argile et à son rapport à la teneur en matières organiques, est un peu plus élevée dans la parcelle 1 de Grignon que dans la parcelle 2 et qu'à Montmirail. Ces 2 dernières ont eu, lors d'observations au champ, un comportement légèrement battant. D'après leur composition, les 3 matériaux ont une aptitude faible à la fissuration ; nos observations montrent cependant que celle-ci n'est pas nulle (cf. § III B-2). Les histoires culturales sont différentes et probablement plus dégradantes dans la parcelle 2 de Grignon et à Montmirail que dans la parcelle 1 de Grignon, en raison de la présence de maïs dans la rotation culturale des 2 premières (MANICHON, $1982 a$ ).

Les caractéristiques climatiques des 4 situations étudiées sont présentées au tableau 2. Les essais de Grignon 1982 et 1983 (notés G82 et G83 dans la suite du texte) se caractérisent par une pluviométrie relativement élevée par rapport à l'évaporation potentielle (ETP, formule de BOUCHET), au contraire de Grignon 1985 (G85). A Montmirail (M82), les précipitations sont relativement faibles ; l'ETP n'a pas été mesurée. Dans tous les cas, la période comprise entre la levée

TABLEAU 1

Caractéristiques des parcelles d'essai.

Characteristics of the experimental fields.

\begin{tabular}{|c|c|c|c|}
\hline & \multicolumn{3}{|c|}{ Parcelles } \\
\hline & $\begin{array}{c}\text { Grignon } 1 \\
\text { (G82) }\end{array}$ & $\begin{array}{c}\text { Grignon } 2 \\
\text { (G83 et G85) }\end{array}$ & $\begin{array}{c}\text { Montmirail } \\
\text { (M82) }\end{array}$ \\
\hline Succession de culture (10 dernières années) & $\begin{array}{l}\text { Rotation } \\
\text { Pois d'hiver } \\
\text { Blé d'hiver }\end{array}$ & Monoculture de maïs & $\begin{array}{c}\text { Rotation } \\
\text { Maiis } \\
\text { Blé d'hiver }\end{array}$ \\
\hline Précédent cultural & Blé d'hiver & Maïs & Blé d'hiver \\
\hline \multicolumn{4}{|l|}{ Granulométrie (couche labourée) } \\
\hline Argile $(<2 \mu \mathrm{m})$ & 23,0 & 18,0 & 23,0 \\
\hline Limon fin $(2-20 \mu \mathrm{m})$ & 23,1 & 24,0 & 23,0 \\
\hline Limon grossier $(20-50 \mu \mathrm{m})$ & 42,8 & 50,4 & 45,6 \\
\hline Sable $(50-200 \mu \mathrm{m})$ & 8,9 & 6,0 & 7,0 \\
\hline Matières organiques & 2,2 & 1,6 & 1,4 \\
\hline Nature pédologique & \multicolumn{2}{|c|}{ Sol brun faiblement lessivé sur loess apparaissant vers $70 \mathrm{~cm}$} & Sol lessivé hydromorphe \\
\hline
\end{tabular}


TABLEAU 2

Données climatiques pour la période levée-3 semaines après floraison du maïs (A Montmirail, les températures et les ETP $n$ 'étaient pas disponibles) flo. : sortie des soies. $7 f$ : stade 7 feuilles visibles. $j$. : jours.

Climatic data for the period emergence - 3 weeks after maize silking. (In Montmirail, temperature and PET datas were not available) flo. : silking. $7 f$ : stage 7 visible leaves. $j$. : day.

\begin{tabular}{|c|c|c|c|c|c|c|c|}
\hline & \multicolumn{4}{|c|}{ Cumuls sur la période levée-3 semaines après floraison } & \multicolumn{3}{|c|}{ Périodes sans pluies } \\
\hline & Dates & $\begin{array}{l}\text { Temp. } \\
\text { cumul. } \\
{ }^{\circ} \mathrm{C}\end{array}$ & $\begin{array}{c}\mathrm{P} \\
\text { cumul. } \\
\mathrm{mm}\end{array}$ & $\begin{array}{c}\text { ETP } \\
\text { cumul. } \\
\text { mm }\end{array}$ & Dates & $\begin{array}{c}\text { Stades } \\
\text { (trait. O) }\end{array}$ & $\begin{array}{c}\text { ETP } \\
\text { cumul } \\
\mathrm{mm}\end{array}$ \\
\hline G82 & $23-5$ au $4-8$ & 893 & 176 & 207 & $\begin{array}{l}4-6 \text { au } 10-6 \\
3-7 \text { au } 14-7\end{array}$ & $\begin{array}{l}4 f \text { à } 6 f \\
4 f \text { à flo. }\end{array}$ & $\begin{array}{l}25 \\
37\end{array}$ \\
\hline M82 & $25-5$ au $6-8$ & - & 139 & - & $\begin{array}{l}2-6 \text { au } 10-6 \\
3-7 \text { au } 22-7\end{array}$ & $\begin{array}{l}4 f \dot{a} 6 f \\
12 f \dot{a} \\
\text { flo. }+6 j\end{array}$ & $\begin{array}{l}- \\
-\end{array}$ \\
\hline G83 & $23-5$ au $6-8$ & 899 & 204 & 244 & $\begin{array}{l}9-6 \text { au } 21-6 \\
7-7 \text { au } 18-7\end{array}$ & $\begin{array}{c}\text { 6f a } 9 f \\
14 f \dot{a} \\
\text { flo. }+2 j\end{array}$ & $\begin{array}{l}43 \\
52\end{array}$ \\
\hline G85 & $17-5$ au $7-8$ & 846 & 123 & 274 & $\begin{array}{l}24-5 \text { au } 14-6 \\
24-6 \text { au } 13-7\end{array}$ & $\begin{array}{l}3 f \text { a } 7 f \\
10 f \dot{d} \\
\text { flo. }-2 j\end{array}$ & $\begin{array}{l}59 \\
67\end{array}$ \\
\hline
\end{tabular}

du maïs et 3 semaines après floraison est formée d'une succession de périodes orageuses à forte pluviométrie et d'épisodes sans pluie pendant lesquels l'ETP était élevée. L'un de ceux-ci s'est produit dans toutes les situations aux environs du stade " 6 feuilles visibles » (noté $6 \mathrm{f}$ ). Une seconde période sans pluie s'est déroulée pendant les semaines précédant la floraison, suivie à $\mathrm{G} 82$ et $\mathrm{G} 83$ d'un orage violent $(72$ et $33 \mathrm{~mm}$ respectivement), au contraire de M82 et G85.

\section{B. Obtention des états structuraux souhaités}

Trois itinéraires techniques (SEBILLOTTE, 1978) ont été employés pour créer les états définis en introduction. Le sol a été labouré à la profondeur habituelle pour cette opération dans les parcelles étudiées, en moyenne $28 \mathrm{~cm}$ avant compactage. En $\mathrm{O}$ et $\mathrm{C}$, le labour a été réalisé en décembre sur sol ressuyé et à vitesse rapide, de façon à obtenir une fragmentation maximale. En $\mathrm{C}$, le sol a été tassé après les gels hivernaux sur la totalité de la surface des parcelles expérimentales de ce traitement au moyen d'un tracteur lourd $(7 \mathrm{~T})$ dont les pneumatiques étaient gonflés à 1,8 bar à l'arrière et 2,5 bars à l'avant. Cette opération a été réalisée à faible vitesse et répétée jusqu'à l'obtention de l'état souhaité. Les parcelles expérimentales de $\mathrm{B}$ ont subi un tassement semblable à celui de $\mathrm{C}$, suivi en avril par un labour en conditions humides et à vitesse lente.

La préparation du lit de semence a été réalisée à l'aide d'une herse animée oscillante, outil agressif qui permet l'obtention d'un horizon $\mathrm{H} 1$ suffisamment affiné pour que la levée soit satisfaisante, quel que soit l'état du sol après labour. La profondeur de ce travail est de $10 \mathrm{~cm}$, mesurés avant foisonnement ; l'épaisseur de l'horizon $\mathrm{H} 5$ est donc de $18 \mathrm{~cm}$ en moyenne. Ce travail a été fait perpendiculairement au sens du labour et du semis. Les emplacements des passages de roues du tracteur portant la herse ont été repérés afin de pouvoir délimiter des placettes mesurant $1,20 \mathrm{~m}$ (dans le sens $\mathrm{du}$ rang) sur $0,80 \mathrm{~m}$ (parallèlement au rang), indemnes de compactage après labour en $\mathrm{O}$ et $\mathrm{B}$. Les mesures et observations sur l'état structural et l'état hydrique du sol ont été effectuées dans ces placettes, de même que celles faites ultérieurement sur le peuplement végétal (cf. article suivant). Une culture de maïs (variété LG1) a été implantée dans tous les essais pendant la première semaine de mai.

\section{Etude de l'état structural}

\section{Etat au semis}

Entre 4 et 8 placettes (tabl. 3) ont été observées immédiatement après semis, pour vérifier que les diffé-

\section{TABLEAU 3}

Nombre de placettes de $0,80 \mathrm{~m}$ de large caractérisées par la méthode de cartographie structurale.

Number of samples $0.80 \mathrm{~m}$ in characterized width, by the structural mapping method.

\begin{tabular}{lccc}
\hline & $\mathrm{O}$ & $\mathrm{B}$ & $\mathrm{C}$ \\
\hline G82 & & & \\
23 au 28-5 & 4 & 4 & 4 \\
20 au 23-7 & 12 & 8 & 8 \\
\hline M82 & & & \\
28 au 31-5 & 4 & 4 & 8 \\
2 au 5-8 & 4 & 4 & 8 \\
\hline G83 & & & \\
23 au 28-5 & 5 & 5 & 5 \\
10 au 13-6 & 4 & 4 & 4 \\
17 au 22-6 & 7 & 4 & 4 \\
$29-6$ au 1-7 & 7 & 4 & 7 \\
$20-7$ au 1-8 & 11 & 12 & 10 \\
\hline G85 & & & \\
17 au 22-5 & 6 & & 6 \\
20 au 28-7 & 4 & & \\
\hline \hline
\end{tabular}


renciations souhaitées avaient été obtenues. Après ouverture d'une fosse, l'état physique de la couche labourée a été caractérisé par la méthode cartographique proposée par MANICHON (1982a), complétée par des mesures de masse volumique et de teneur en eau du sol. Après avoir délimité les différents horizons sur la face verticale de la fosse, nous avons distingué au sein de chaque horizon des unités morphologiques dont l'état structural est défini par l'appréciation de 2 niveaux d'organisation structurale :

- L'état interne des éléments structuraux : continu et de porosité visible très réduite (noté $\Delta$ ), ou plus poreux (noté $\Gamma$ ), états différenciés par la morphologie des faces de rupture des mottes. Ces observations ont été confrontées à des mesures de masse volumique sèche d'une trentaine de mottes de chaque type pour chaque essai. La procédure suivie pour ces mesures est celle décrite par STENGEL (1984).

- L'assemblage des éléments structuraux, pour lequel les modalités suivantes ont été retenues : état massif (noté $\mathrm{M}$ ) où une unité morphologique est constituée d'un seul élément structural ; état fragmentaire (F) caractérisé par la dimension des éléments qui le constituent : terre fine (tf), mottes centimétriques (pm) ou décimétriques $(\mathrm{gm})$. Les cavités associées à ces unités morphologiques sont notées $V$, les états massifs fissurés sont notés MR.

Après que ses contours ont été cartographiés, chaque unité morphologique de la carte structurale est donc renseignée par les symboles caractérisant le mode d'arrangement des mottes, et l'état interne de celles-ci. Des exemples de cartes figurent dans un article précédent (TARDIEU \& MANICHON, 1986).

Des mesures de masse volumique du sol ont été faites à une échelle décimétrique au moyen d'une sonde à atténuation de rayons $\gamma$ (SOANE et al., 1971), modèle ELCOMATIC (écartement entre pals : $12 \mathrm{~cm}$ ).

\section{Observations au cours de la campagne}

En 1983, les cartographies de l'état structural ont été répétées plusieurs fois, à des dates choisies en fonction des stades phénologiques des peuplements végétaux (cf. TARDIEU \& MANICHON, 1987 à paraître). La teneur en eau du sol a été mesurée à G83 et G85 au début et à la fin de chaque période sans pluie. Dans la couche labourée, 8 à 16 échantillons de $70 \mathrm{~cm}^{3}$ environ ont été prélevés dans les couches $3-10,10-15$ et 15 $28 \mathrm{~cm}$, chaque échantillon étant également repéré par l'état structural de l'unité morphologique d'où il provient. Dans les couches non travaillées, la teneur en eau a été suivie par humidimétrie neutronique (TARDIEU, 1987, à paraître).

\section{RÉSULTATS}

\section{A. Etat structural : observations au semis}

\section{Etat des couches non travaillées}

La comparaison des masses volumiques du sol dans la couche $30-50 \mathrm{~cm}$ (tabl. 4) montre que, dans les situations G82, M82 et G83, les différences entre trai-

\section{TABLEAU 4}

Masses volumiques sèches $\left(\mathrm{g} . \mathrm{cm}^{-3}\right)$ mesurées avec la sonde $\gamma$ dans la couche 30 à $50 \mathrm{~cm} . \bar{x}$ : moyenne; s : écart-type. A l'intérieur d'une ligne, les valeurs suivies par une lettre commune ne diffèrent pas dans un test Fisher à niveau de risque 5 p. 100.

Dry bulk densities $\left(\mathrm{g} . \mathrm{cm}^{-3}\right)$ measured with the $\gamma$ ray probe in the 30 $50 \mathrm{~cm}$ layer. $\bar{x}:$ mean; $s:$ standard deviation. Within a line, values followed by a common letter do not differ at the $5 \%$ level according to the Fisher test.

\begin{tabular}{|c|c|c|c|}
\hline & \multicolumn{3}{|c|}{ Traitements } \\
\hline & $\mathrm{O}$ & B & $\mathrm{C}$ \\
\hline \multicolumn{4}{|l|}{ G82 } \\
\hline $\bar{x}$ & 1,49 & 1,46 & 1,47 \\
\hline$s$ & 0,04 & 0,02 & 0,06 \\
\hline Test & $\mathrm{a}$ & $\mathrm{a}$ & $\mathrm{a}$ \\
\hline \multicolumn{4}{|l|}{ M82 } \\
\hline $\bar{x}$ & 1,49 & - & 1,49 \\
\hline $\mathrm{S}$ & 0,06 & - & 0,08 \\
\hline Test & $a$ & - & a \\
\hline \multicolumn{4}{|l|}{ G83 } \\
\hline $\bar{x}$ & 1,43 & 1,38 & 1,42 \\
\hline$s$ & 0,05 & 0,06 & 0,04 \\
\hline Test & $\mathrm{a}$ & $\mathrm{a}$ & $\mathrm{a}$ \\
\hline
\end{tabular}

tements sont très faibles et non significatives. Les tassements en $\mathrm{B}$ et $\mathrm{C}$ n'ont donc pas modifié la masse volumique de cette couche. Ces mesures sont confirmées par les observations morphologiques : les pores visibles de la couche $30-50 \mathrm{~cm}$, galeries d'origine animale et fissures, n'ont pas été détruits en $B$ et $C$. Les mesures de masse volumique n'ont pu être répétées à G85, mais les observations morphologiques fournissent les mêmes résultats que celles des années précédentes.

\section{Types de mottes rencontrées dans l'horizon $\mathrm{H5}$ (tabl. 5 et 6)}

L'essai G82 est le seul où les différences entre traitements portent sur l'état interne des mottes. En $O$ et $C$, la quasi-totalité des mottes observées sont de type $\Gamma$. Au contraire, à M82, G83 et G85, seules des mottes $\Delta$ ont été trouvées.

Les mesures de densité sèche de mottes montrent (tabl. 5) qu'il existe une relation étroite entre les caractéristiques morphologiques $\Delta$ ou $\Gamma$ et les densités. On peut noter que les moyennes et les gammes de variation des densités de mottes $\Delta$ sont semblables en G83 et G85, essais situés sur la même parcelle, et peu différentes de celles de G82 situées sur une parcelle voisine. Les densités de mottes mesurées à Montmirail sont inférieures à celles mesurées à Grignon. Il est probable que cela est dû à des densités texturales (FIES \& STENGEL, $1981 a$ ) différentes entre parcelles.

\section{Etat de l'horizon H5 au semis (tabl. 6)}

\section{a) A l'intérieur de chaque situation expérimen- tale}

La comparaison des masses volumiques mesurées avec la sonde $\gamma$ montre qu'il existe une différence significative entre $\mathrm{O}$ et $\mathrm{C}$. Cette mesure n'a été faite en $\mathrm{B}$ 
TABLEAU 5

Moyenne et gamme de variation des masses volumiques sèches pour les mottes de type $\Delta$ et $1: \bar{x}:$ moyenne. Average and range of dry bulk densities for clods of $\Delta$ and $\Gamma$ types. $\bar{x}$ : average.

\begin{tabular}{|c|c|c|c|c|c|c|}
\hline & \multicolumn{6}{|c|}{ Types de mottes } \\
\hline & \multicolumn{3}{|c|}{$\Delta$} & \multicolumn{3}{|c|}{$\Gamma$} \\
\hline & $\mathrm{n}$ & $\overline{\mathrm{x}}$ & Gamme de variation & $\mathrm{n}$ & $\bar{x}$ & Gamme de variation \\
\hline G82 & 18 & 1,77 & 1,70 à 1,85 & 15 & 1,60 & 1,56 à 1,70 \\
\hline M82 & 28 & 1,73 & 1,68 à 1,79 & & & \\
\hline G83 & 48 & 1,82 & 1,72 à 1,90 & & & \\
\hline G85 & 35 & 1,83 & 1,75 à 1,85 & & & \\
\hline
\end{tabular}

TABLEAU 6

Caractéristiques de l'horizon H5 au semis. $\bar{x}:$ moyenne; $i$ : intervalle de confiance à 0,05 . $H 5$ layer characteristics at planting. $\bar{x}$ : average ; $i$ : confidence interval at 0.05 .

\begin{tabular}{|c|c|c|c|c|c|c|c|}
\hline & \multicolumn{4}{|c|}{$\begin{array}{l}\text { Pourcentage de surface du profil occupé } \\
\text { par les différents états structuraux }\end{array}$} & \multirow[t]{2}{*}{$\begin{array}{c}\text { Etat interne dominant } \\
\text { des mottes }\end{array}$} & \multicolumn{2}{|c|}{$\begin{array}{l}\text { Masse volumique moyenne } \\
\text { de l'horizon }\end{array}$} \\
\hline & M & MR & FVgm & $\mathrm{Ftf}+\mathrm{pm}$ & & $\bar{x}$ & $\mathrm{i}$ \\
\hline \multicolumn{8}{|c|}{ Traitement $\mathrm{O}$} \\
\hline G82 & 10 & 0 & 8 & 82 & $\Gamma$ & 1,41 & 0,02 \\
\hline M82 & 28 & 0 & 25 & 47 & $\Delta$ & 1,45 & 0,01 \\
\hline G83 & 17 & 0 & 26 & 57 & $\Delta$ & 1,30 & 0,03 \\
\hline G85 & 24 & 0 & 21 & 55 & $\vec{\Delta}$ & 1,33 & 0,06 \\
\hline \multicolumn{8}{|c|}{ Traitement B } \\
\hline G82 & 34 & 8 & 47 & 11 & $\Delta$ & 1,43 & 0,01 \\
\hline M82 & 37 & 7 & 50 & 6 & $\Delta$ & - & - \\
\hline G83 & 43 & 7 & 40 & 10 & $\bar{\Delta}$ & - & - \\
\hline \multicolumn{8}{|c|}{ Traitement C } \\
\hline G82 & 98 & 2 & 0 & 0 & $\Gamma$ & 1,55 & 0,01 \\
\hline M82 & 95 & 5 & 0 & 0 & $\Delta$ & 1,63 & 0,01 \\
\hline G83 & 100 & 0 & 0 & 0 & $\Delta$ & 1,69 & 0,02 \\
\hline G85 & 100 & 0 & 0 & 0 & $\Delta$ & 1,64 & $0,03$. \\
\hline
\end{tabular}

M : état massif ; MR : état massif fissuré ; FVgm : état fragmentaire mottes décimétriques et cavités structurales ; Ftf + pm : état fragmentaire : terre fine et mottes centimétriques.

qu'à G82 ; elle ne permet pas de mettre en évidence de différence avec le traitement $O$.

En revanche la comparaison des pourcentages de surface de l'horizon $\mathrm{H} 5$ occupé par les différents états structuraux montre des différences significatives entre les 3 traitements (test du $\chi^{2}$ ). On observe la prédominance des états fragmentaires fins ( $F$ tf) en $O$, des mottes et des cavités structurales en B ( $\mathrm{M}$ et FV gm), et des états massifs en $\mathrm{C}$.

La porosité structurale (partie non texturale de la porosité totale, STENGEL, 1979) est donc nettement supérieure en $\mathrm{O}$ par rapport à $\mathrm{C}$; elle est, au contraire, proche en $\mathrm{O}$ et $\mathrm{B}$ en 1982, mais répartie différemment : nombreux pores millimétriques en $O$, cavités centimétriques peu nombreuses en $\mathrm{B}$.

\section{b) Entre situations expérimentales}

La comparaison entre états obtenus pour un même traitement ne peut être faite en considérant les mesures de masse volumique du sol, puisque les densités texturales sont probablement différentes en liaison avec la granulométrie. Les observations cartographiques mon- trent que les proportions de l'horizon H5 correspondant aux différentes catégories structurales sont stables d'une situation à l'autre dans le traitement B. Le traitement $\mathrm{O}$ apparaît plus variable entre situations. En particulier, une proportion notable (environ $20 \mathrm{p}$. 100) d'états fragmentaires grossiers n'est observée que lorsque l'état interne dominant des mottes est de type $\Delta$ (M82, G83 et G85). D'autre part, l'état interne des mottes du traitement $C$ est $\Gamma$ à G82, et $\Delta$ dans les autres champs expérimentaux. Le tassement a donc été moins intense à G82.

\section{B. Evolution au cours de la campagne}

\section{Teneur en eau du sol (tabl. 7)}

Les valeurs mesurées au semis, à G83 et G85, sont peu différentes entre elles, et également très proches des humidités mesurées au cours d'événements faiblement pluvieux au début du printemps. Elles peuvent donc être considérées comme les humidités à la « capacité au champ ». Dans l'horizon $H 1$, les valeurs 
TABLEAU 7

Humidité pondérale (p. 100) à la " capacité au champ " et variations d'humidité au cours de la première période sans pluie de 1983. $\bar{x}$ : moyenne ; $s$ : écart-type.

Soil water content by weight (\%) at « field capacity » and variation of water content during the first dry period of 1983. $\bar{x}$ : average; $s$ : standard deviation.

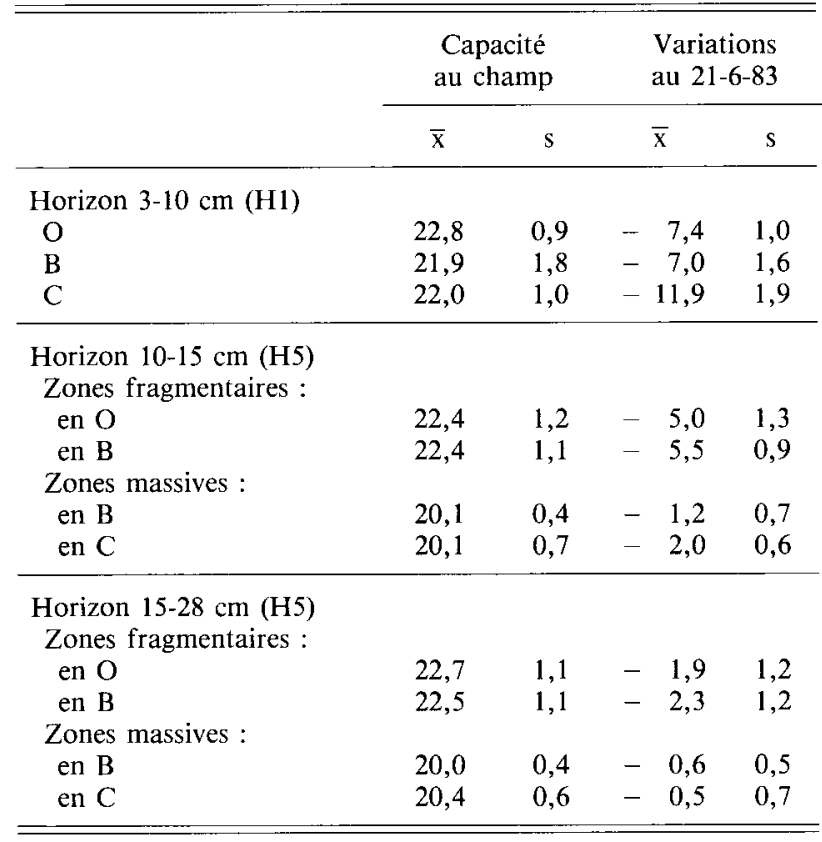

moyennes d'humidité pondérale correspondant à chaque traitement sont proches les unes des autres, aux environs de 22 p. 100 , et la variabilité intra traitement est faible. Dans l'horizon $\mathrm{H} 5$, elles sont légèrement plus faibles dans les zones massives de $\mathrm{B}$ et $\mathrm{C}$ que dans les zones fragmentaires ( 20 contre 22 p. 100), ce qui est cohérent avec les travaux de PAPY (1984) et de CURMI (1985). Dans les couches non travaillées, les humidités sont comprises entre 18 et 20 p. 100 . Elles sont restées stables jusqu'au début de la $1^{\text {re }}$ période sans pluie. Au cours de celle-ci, l'humidité de la couche labourée s'est abaissée dans les zones fragmentaires de $\mathrm{O}$ et $\mathrm{B}$, mais a relativement peu varié dans les zones massives de $B$ et C. L'épisode pluvieux suivant a ramené rapidement la couche labourée à la capacité au champ, avant un nouveau dessèchement du sol consécutif à la seconde période sans pluie. Des phénomènes semblables à ceux de la $1^{\text {re }}$ période ont alors été observés ; ces faits seront analysés dans le $3^{\mathrm{e}}$ article de cette série (TARDIEU, à paraître).

\section{Etat structural (tabl. 7)}

En l'absence de toute opération culturale entre le semis et la floraison, la seule cause d'évolution de l'état structural de $\mathrm{H} 5$ a été la variation de teneur en eau du sol. Dans le traitement $O$, cette évolution structurale a été relativement peu importante : les proportions de chaque type d'état structural sont peu différentes entre la levée et la floraison. En B, on note une fissuration des zones massives : un état massif fissuré MR a été observé à la floraison, la proportion de zones fragmentaires restant stable. Cette fissuration a été encore plus nette en $\mathrm{C}$ : à la suite de la première
TABLEAU 8

Caractéristiques de l'horizon HS à la floraison. H5 layer characteristics at flowering.

\begin{tabular}{|c|c|c|c|c|c|}
\hline & \multicolumn{5}{|c|}{$\begin{array}{l}\text { Pourcentage de surface du profil occupé } \\
\text { par les différents états structuraux }\end{array}$} \\
\hline & $\mathbf{M}$ & MR & FVgm & $\begin{array}{l}\text { Ftf } \\
+\mathrm{pm}\end{array}$ & $\begin{array}{l}\text { Fentes } \\
\text { retrait }\end{array}$ \\
\hline \multicolumn{6}{|c|}{ Traitement $\mathrm{O}$} \\
\hline G82 & 10 & 0 & 5 & 85 & 0 \\
\hline M82 & 30 & 0 & 24 & 46 & 0 \\
\hline G83 & 17 & 1 & 16 & 66 & 0 \\
\hline G85 & 8 & 9 & 22 & 61 & 0 \\
\hline \multicolumn{6}{|c|}{ Traitement B } \\
\hline G82 & 19 & 20 & 58 & 3 & 0 \\
\hline M82 & 26 & 18 & 49 & 7 & 0 \\
\hline G83 & 37 & 11 & 50 & 2 & 0 \\
\hline \multicolumn{6}{|c|}{ Traitement C } \\
\hline G82 & 65 & 20 & 0 & 0 & 15 \\
\hline M82 & 69 & 23 & 0 & 0 & 8 \\
\hline G83 & 87 & 8 & 0 & 0 & 5 \\
\hline G85 & 80 & 14 & 0 & 0 & 6 \\
\hline
\end{tabular}

période sans pluie de 1983 (observation du 26/06), des fentes de retrait sont apparues dans l'horizon H5. Le 4/07, celles-ci traversaient totalement cet horizon. Elles ont été également observées à la floraison dans les 3 autres situations. La maille de cette fissuration étant de l'ordre du décimètre, l'horizon H5 du traitement C était donc constitué à la floraison d'éléments structuraux prismatiques, de dimensions décimétriques, sépa rés par des fentes de retrait subverticales.

\section{Analyse de la variabilité entre placettes à la floraison}

L'effectif relativement important des placettes observées à la floraison a permis une étude de la variabilité entre placettes, destinée à évaluer les parts respectives des variabilités intra traitement, inter traitements et inter champs expérimentaux de l'état structural. Nous avons étudié pour cela, par analyse factorielle des correspondances, le tableau à 8 variables et 79 individus formés par les proportions de surface de l'horizon H5 occupé par les 8 types d'états structuraux rencontrés, pour chacune des placettes étudiées à la floraison.

Si l'on considère comme seuil pour l'interprétation des axes une inertie égale à l'inverse du nombre de variables (CANEILL \& DANZART, 1984), 3 axes contribuent à rendre compte de la dispersion des données. Le premier, qui porte $36 \mathrm{p} .100$ de l'inertie totale du nuage, est constitué par l'opposition entre les variables $\mathrm{M} \Delta$ et $\mathrm{FV}$ gm d'une part, $\mathrm{F}$ tf et $\mathrm{M}$ d'autre part. Le deuxième (26 p. 100 d'inertie) oppose $\mathrm{M} \Delta$ et « Fentes de retraït 》 à $F$ tf. Le dernier (16 p. 100 d'inertie) oppose $\mathrm{M} \Delta$ à $\mathrm{FV}$ gm. Ces axes différencient 4 nuages disjoints de points :

- Les états B de G82, M82 et G83, dont les individus sont très regroupés, sont en correspondance avec les variables $\mathrm{FV}$ gm et $\mathrm{M} \Delta$. 
axe $226 \%$ inertie

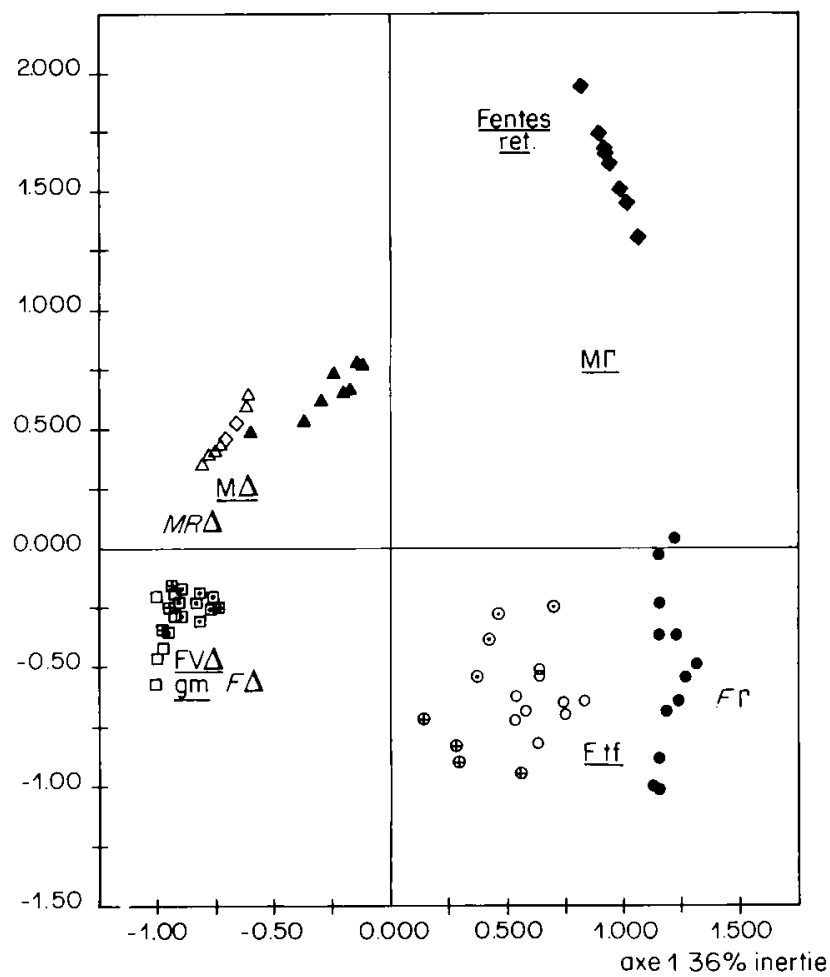

$\begin{array}{ll}\text { Tralt. } & \\ \text { G82 } & 0 \\ \text { Mez } & 0 \\ 683 & 0 \\ \text { Ge5 }\end{array}$

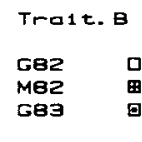

Trast.c

Gez

MB2

G93

Figure 1

Résultat de l'AFC: axes l et 2. Les variables ayant une contribution absolue supérieure à $12,5 \%$ (inverse du nombre de variables) sur l'un de ces axes sont figurées en caractères normaux et soulignés, les autres en italiques. Tous les individus représentés ont une somme de contributions relatives sur les 2 premiers axes supérieure à $40 \%$.

Results of the factor correspondence analysis : 1 st and 2 nd axes. The variables having an absolute contribution greater than $12.5 \%$ (inverse of variables number) on one of these axes are figured with normal underlined characters, the others in italics. All presented individuals had a sum of relative contributions greater than $40 \%$ on the 2 presented axes.

- Les états C de M82, G83 et G85 en correspondance avec la variable $\mathrm{M} \Delta$ et « Fentes de retrait». Ces 2 nuages, déjà distincts sur le plan formé des 2 premiers axes, le sont aussi sur le $3^{\mathrm{e}}$ axe.

- L'état C de G82, lié aux variables MГ et « Fentes de retrait $\gg$.

- L'état $O$, dont la relative dispersion est causée par des différenciations entre situations : un sous-nuage correspond aux situations où l'état des mottes est de type $\Delta(M 82, G 83$ et G85), et l'autre où il est de type $\Gamma$ (G82).

\section{DISCUSSION}

\section{A. Caractéristiques et représentativité des états créés}

1. Les itinéraires techniques appliqués aux parcelles expérimentales n'ont différencié entre traitements qu'une couche de sol (H5) relativement peu épaisse : celle comprise entre le fond du travail superficiel et le fond du labour. Ces $18 \mathrm{~cm}$, environ, doivent être comparés à la profondeur potentielle d'enracinement du maïs, souvent voisine du $\mathrm{m}$. Les états $\mathrm{O}$, B et $\mathrm{C}$ ne concernent donc qu'une faible proportion $\mathrm{du}$ volume atteint par les racines. Des effets relativement superficiels des tassements agricoles avaient déjà été trouvés par VOORHEES et al. (1978), SOANE et al. (1982) et GUERIF (1984) : après tassements, ces auteurs n'ont pas observé de variation de masse volumique au-delà d'une profondeur d'environ $30 \mathrm{~cm}$.

Les mottes $\Delta$ subissent au cours de la campagne une modification importante d'état structural : leur fissuration, qui peut être datée au début du mois de juillet pour G83, provoque une variabilité spatiale très importante de la résistance à la pénétration des racines dans cet horizon. Il est à noter que cette fissuration a été observée alors que l'aptitude du matériau pouvait être considérée comme faible et qu'elle survient dans les parcelles expérimentales où la dessiccation est la moins rapide (tabl. 8). L'hypothèse la plus vraisemblable pour expliquer ce fait nous semble être celle suggérée par les travaux de TRI (1968) : les racines, pénétrant dans les amorces de fentes de retrait, provoquent une dessiccation localisée autour de celles-ci. Un retrait volumique s'ensuit qui, bien que de faible amplitude, provoque des ruptures dans la zone tassée et permet aux racines de progresser. Le phénomène s'autoaggrave donc à partir d'amorces de discontinuités, pour aboutir à une transformation importante des caractéristiques mécaniques de l'horizon H5.

2. Des états structuraux de la couche labourée semblables à ceux définis et obtenus ici en expérimentation, ont été observés à plusieurs reprises lors d'enquêtes en parcelles d'agriculteurs (SEBILLOTTE, 1963; MANICHON \& SEBILlOTTE, 1975 ; MEYNARD et al., 1981 ; PELlERIN, 1984 ; Enquêtes « Maïs progrès » LIMAGRAIN, non publié). Dans des parcelles limoneuses du Béarn, soumises à monoculture de maïs, MANICHON (1985) les a reliés aux itinéraires techniques utilisés par les agriculteurs et aux conditions hydriques du sol lors des passages d'outils. Les états de type B sont observés quand le sol a été intensément tassé avant le labour, par exemple lors de la récolte du précédent cultural ou lors d'épandages de lisier. Les états de type $C$ correspondent à des tassements répétés en conditions hydriques défavorables lors des travaux post-labour. Ils sont, notamment, observés dans les «fourrières 》 des parcelles, et sur l'ensemble de celles-ci lorsque le travail superficiel est effectué au moyen d'outils étroits et peu agressifs, nécessitant un nombre important de passages. Des états proches de C ont d'autre part été observés au champ lorsqu'un semis direct a été effectué sur un sol préalablement tassé. Les états B et C correspondent donc à des situations existant réellement $\mathrm{au}$ champ, alors qu'il est rare que les états $O$ soient observés sur une proportion importante du profil cultural. Compte tenu de la charge des calendriers de travaux, il est peu fréquent que l'ensemble des travaux agricoles puisse être effectué par les agriculteurs dans des conditions hydriques telles qu'aucun tassement ne s'ensuive (PAPY, 1984) : des 3 états que nous avons introduits dans cette expérimentation, l'état $O$ n'est vraisemblablement pas le plus fréquent au champ. 


\section{B. Reproductibilité et utilisation comme traitements expérimentaux des états créés}

1. Les états $\mathrm{O}, \mathrm{B}$ et $\mathrm{C}$ sont suffisamment stables entre situations pour qu'il soit acceptable de désigner sous le même vocable des états obtenus lors d'années différentes, dans plusieurs localités.

Cependant nous n'avons réussi à contrôler que le mode d'arrangement des mottes, et non leur état interne. Lorsque celui-ci était de type $\Delta$ au début de l'essai, il n'a pas été possible de le transformer car cette opération nécessite en général plusieurs années (MANICHON, 1982b). Dans les états O de M82, G83 et G85, les mottes étaient donc de type $\Delta$, ce qui a joué sur leur taille. Cette interaction entre état interne et mode d'arrangement des mottes avait déjà été notée par MANICHON (1982a). Inversement, à G82, l'état initial des mottes était de type $\Gamma$; les tassements appliqués en $C$ n'ont pas été suffisants pour détruire la porosité intramottière : l'état des mottes n'a pas été modifié.

Il nous paraît donc nécessaire de renseigner les types d'état de la couche labourée par l'état interne des mottes: la désignation des états obtenus serait donc « $\mathrm{O} \Delta$ » à M82, G83 et $\mathrm{G} 85 ;$ « $\mathrm{O} \Gamma$ » à $\mathrm{G} 82 ;$ « $\mathrm{B} \Delta$ » dans toutes les situations étudiées ; « $\mathrm{C} \Gamma$ » à $\mathrm{G} 82$ et " $\mathrm{C} \Delta$ " à M82, G83 et G85.

2. Ces états ne pourront être considérés comme traitements dans les expérimentations présentées dans les prochains articles, que parce qu'il existe une disjonction complète entre les placettes de traitements différents. L'AFC a montré qu'aucune placette appartenant à un traitement n'a les caractéristiques d'un autre traitement. Nous pensons que cette règle de disjonction, plus sévère que la simple signification statistique, est nécessaire pour l'interprétation des essais sur le travail du sol. Elle a requis de notre part des précautions plus grandes que celles qui sont prises dans la majorité des expérimentations sur le travail du sol, comme le piquetage de chacune des traces de roues lors de la réalisation du travail superficiel et du semis.

\section{CONCLUSION}

Les résultats présentés montrent qu'il a été possible de modéliser sur un dispositif expérimental 3 états structuraux de la couche labourée qui avaient été souvent observés en parcelles cultivées : les états types $O$, $B$ et $C$, tous issus d'un labour, ont été obtenus en jouant sur des compactages avant ou après le labour et sur la date de ce dernier. La variabilité spatiale de chacun, à l'échelle métrique, est suffisamment maîtrisée pour qu'il soit possible de les considérer comme des traitements expérimentaux lors d'études du comportement hydrique de la couche labourée (PAPY, 1984), ou des conséquences de l'état structural du sol sur l'enracinement, l'alimentation hydrique et la croissance de peuplements végétaux (articles suivants de cette série). Les états modélisés ont, d'autre part, été reproductibles dans 3 champs expérimentaux lors d'années différentes, ce qui permet à ces études d'être multilocales ou multiannuelles.

Par rapport aux états modélisés au laboratoire pour étudier les relations entre l'état structural et la pénétration des racines, ceux présentés ici ont 2 caractéristiques importantes : (1) dans nos études, seule une faible fraction du volume potentiellement colonisé par les racines est affectée par les tassements ; ceci n'est généralement pas le cas au laboratoire ; (2) l'état structural de ce volume de sol varie au cours du temps en raison de la fissuration des zones massives. Les états du sol modélisés sont donc définis par des états initiaux, auxquels sont reliées des dynamiques structurales ; cellesci dépendent des caractéristiques pédologiques et climatiques du lieu étudié.

Bien que les états $\mathrm{O}, \mathrm{B}$ et $\mathrm{C}$ représentent des situations existant réellement en parcelles d'agriculteurs, ils ne peuvent rendre compte de la totalité de celles-ci. Nous n'avons, en particulier, pas fait varier l'épaisseur des horizons $\mathrm{H} 1$ et $\mathrm{H} 5$, ni introduit de "semelle de labour ». Ces états ne prennent pas non plus en compte la situation, fréquente au champ, où des traces de roues disjointes existent dans la couche labourée. Une grande gamme d'états structuraux reste donc à explorer.

Recu le 25 novembre 1985. Accepté le 20 novembre 1986.

\section{REMERCIEMENTS}

Les auteurs remercient $\mathbf{J}$. TroIzIER et l'équipe du Centre expérimental de Grignon pour le soin apporté aux travaux agricoles nécessaires à l'obtention des états structuraux souhaités.

\section{RÉFÉRENCES BIBLIOGRAPHIQUES}

Barber S. A., 1971. Effect of the tillage practice on corn root distribution and morphology. Agron. J., 63, 724-726.

Bodet J. M., Nolot J. M., Perroy J., Fourbet J. F., 1976. Présentation des essais. Colloque "Simplification du travail du sol en production céréalière », 23-71. ITCF, Paris, $365 \mathrm{p}$

Boiffin J., Sebillotte M., Couvreur F., 1976. Incidence de la simplification du travail du sol sur l'élaboration du rendement du blé et du maïs. Colloque «Simplification du travail du sol en production céréalière », 239-280. ITCF, Paris, $365 \mathrm{p}$.

Caneill J., Danzart M., 1984. Agronomie et analyse des données. Cycle Supérieur d'Agronomie, INA-PG, Paris (à paraître).
Curmi P., 1985. Comportement physique intrinsèque de mottes à macroporosité différente. Séminaire "Land and Water Use and Management ». CEE-Avignon, 17-18/09/85, à paraître.

Ellis F. B., Barnes B. T., 1978. Effects of cultivation on the root development of winter cereals in three clay soils. Letcombe Lab. anu. Rep., 1977, 36-37.

Fies J. C., Stengel P., 1981. Densité texturale de sols naturels. I. - Méthodes de mesure. Agronomie, 1, 651-658. Il. - Eiéments d'interprétation. Agronomie, 1, 659-666.

Guerif J., 1984. The influence of water-content gradient and structure anisotropy on soil compressibility. J. agric. Enging. Res., 29, 367-374. 
Henin S., Gras R., Monnier G., 1969. Le Profil Cultural. Masson, Paris, $332 \mathrm{p}$.

Manichon H., 1982a. Influence des systèmes de culture sur le profil cultural: Elaboration d'une méthode de diagnostic basée sur l'observation morphologique. Thèse Doct. Ing., INA-PG, Paris, $214 \mathrm{p}$.

Manichon H., 1982b. L'action des outils sur le sol : appréciation de leurs effets par la méthode du profil cultural. Sci. Sol, 203-219.

Manichon H., 1986. Soil-tool interactions : field studies using soil profile descriptions. Sci. Sol., 24, 23-24.

Manichon H., Bodet J. M., 1976. Incidence de la simplification du travail du sol sur l'évolution des profils culturaux. Colloque « Simplification du travail du sol en production céréalière », 109-131. ITCF, Paris, 365 p.

Manichon H., Sebillotte M., 1975. Analyse et prévision des conséquences des passages successifs de différents outils sur le profil cultural. Bull. tech. Inf., 302-303, 569-577.

Meynard J. M., Boiffin J., Caneill J., Sebillotte M., 1981. Elaboration du rendement et fertilisation azotée du blé d'hiver en Champagne Crayeuse. II. - Types de réponse à la fumure azotée et application de la méthode du bilan prévisionnel. Agronomie, 1, 795-806.

Monnier G., Stengel P., 1982. La composition granulométrique des sols : un moyen de prévoir leur fertilité physique. Bull. tech. Inf., 370, 503-512.

Papy F., 1984. Comportement du sol sous l'action des façons de reprise d'un labour au printemps. Effet des conditions climatiques et de l'état structural. Thèse Doct. Ing., INA-PG, Paris, 232 p.

Pellerin S., 1984. Etude des pratiques de travail du sol dans les exploitations du Marais Poitevin. Thèse Doct. Ing., INA-PG, Paris, $165 \mathrm{p}$.

Sebillotte M., 1963. Action de la structure du sol sur une culture de féverole. C. R. Acad. Agric. Fr., 49, 906-914.
Sebillotte M., 1978. Itinéraires techniques et évolution de la pensée agronomique. C. R. Acad. Agric. Fr., 11, 906-914.

Soane B. D., Campbell D. J., Herkes S. M., 1971. Hand held gamma ray equipment for measurement of soil bulk density in the field. J. agric. Eng. Res., 16, 146-156.

Soane B. D., Dickson J. W., Campbell D. J., 1982. Compaction by agricultural vehicles : a review. Soil Tillage Res., 2, 3-36.

Stengel P., 1979. Utilisation de l'analyse des systèmes de porosité pour la caractérisation des systèmes de porosité in situ. Ann. agron., 30, 27-51.

Stengel P., 1984. Analyse de la porosité. C. R. Séminaire CEE Agrimed, 14-18/03/1983, I.N.R.A.-Station Science du Sol d'Avignon, $54 \mathrm{p}$.

Tardieu. F., 1984. Etude au champ de l'enracinement du mais. Influence de l'état structural sur la répartition des racines. Conséquences pour l'alimentation hydrique. Thèse Doct. Ing., INA-PG, Paris, $232 \mathrm{p}$.

Tardieu F., Manichon H., 1986. Caractérisation en tant que capteur d'eau de l'enracinement du maïs en parcelle cultivée. II. - Une méthode d'étude de la répartition verticale et horizontale des racines. Agronomie, 6, 415-425.

Tardieu F., Manichon H., 1987. Etat structural, enracinement et alimentation hydrique du maïs. II. - Croissance et disposition spatiale du systeme racinaire. Agronomie, 7 (3).

Tardieu F., 1987. Etat structural, enracinement et alimentation hydrique du maïs. III. - Disponibilité des réserves en eau du sol. Agronomie, 7 (4).

Tri B. H., 1968. Dynamique de la granulation du sol sous prairie. Ann. agron., 19, 415-439.

Voorhees W. B., Senst C. G., Nelson W. W., 1978. Compaction and soil structure modification by wheel traffic in the northern corn belt. Soil Sci. Soc. Am. J., 42, 344-349. 